\title{
Hepatoprotective effects of Lactococcus chungangensis CAU 1447 in alcoholic liver disease
}

\author{
YoHan Nam, Jong-Hwa Kim, Maytiya Konkit, and Wonyong Kim* $\odot$ \\ Department of Microbiology, Chung-Ang University College of Medicine, Seoul 06974, Republic of Korea
}

\begin{abstract}
Alcoholic liver disease (ALD) is correlated with alcohol consumption, and ALD progression depends on various factors. Some lactic acid bacteria (LAB) are beneficial for mitigating ALD. However, the valuable effects of LAB-derived dairy products remain unclear. Here, we evaluated the effects of Lactococcus chungangensis CAU 1447 dry cells (CAU 1447) and cream cheese derived from CAU 1447 on ALD progression following long-term alcohol consumption in rats. Oral administration of CAU 1447 and CAU 1447 cream cheese significantly reduced alkaline phosphatase, aspartate aminotransferase, alanine aminotransferase, and triglyceride levels. We found that CAU 1447 and CAU 1447 cream cheese downregulated mRNA encoding various cytokines and antioxidative factors in the liver. Oral CAU 1447 cream cheese administration increased short-chain fatty acid, butyrate, and acetate levels in feces. Thus, administration of CAU 1447 and CAU 1447 cream cheese induced hepatoprotective effects, indicating potential applications as a supplement for ALD mitigation.
\end{abstract}

Key words: alcoholic liver disease, Lactococcus chungangensis CAU 1447, cream cheese, toll-like receptor, antioxidant activity

\section{INTRODUCTION}

Alcoholic liver disease (ALD) occurs following excessive consumption of alcohol and is a leading cause of liver cirrhosis-related deaths, accounting for $0.9 \%$ of all global deaths (Rehm et al., 2013). Alcohol overconsumption and long-term intake not only cause liver damage but also disrupt the small intestinal mucosa related to absorption of nutrients and alter the functions of other organs, including the heart, brain, kidney, and liver

Received May 1, 2019

Accepted July 25, 2019.

*Corresponding author: kimwy@cau.ac.kr
(O'Shea et al., 2010; Shukla et al., 2018). The pathogenesis of ALD is associated with alcohol metabolism in the liver, which affects various pathways, including free radical formation, sequential oxidative stress, and inflammatory cytokine secretion pathways (Gyamfi and Wan, 2010; Liu et al., 2014). Toll-like receptor-4 (TLR4) is an important functional receptor that has major roles in the progression of ALD and is associated with nuclear factor (NF)- $\kappa$ B activation (Medzhitov et al., 1997; Shukla et al., 2018). Studies have found that NF- $\kappa \mathrm{B}$ affects proinflammatory cytokine signaling and related mechanisms, including secretion of other inflammatory mediators, such as cyclooxygenase-2 (COX-2), inducible nitric oxide synthase (iNOS), and superoxide dismutase (SOD; Zhao et al., 2017). Some studies have shown that inactivation of NF- $\mathrm{BB}$ suppresses the expression of iNOS, COX-2, and SOD in liver disease (Suetsugu et al., 2005). However, the pathogenesis of ALD is still incompletely understood.

Some lactic acid bacteria (LAB) have been previously shown to exhibit ameliorative effects on ALD and liver injury by suppression of proinflammatory mediators and reduction of alanine aminotransferase (ALT), aspartate aminotransferase (AST), gamma-glutamyl transferase, lactate dehydrogenase, and total bilirubin levels in animal models (Barone et al., 2016; Suo et al., 2016; Lyu et al., 2018). Further studies have shown that heat-killed Lactobacillus strains reduce liver injury induced by alcohol in vitro and in vivo, although some strains cannot inhibit ALT levels (Segawa et al., 2008; Chuang et al., 2016). Lactococcus species are representative starter bacteria for making dairy-based foods, such as cheese and yogurt (Ruggirello et al., 2018). Cream cheese is considered an advantageous carrier to transport live probiotics into the gastrointestinal tract for eventual proliferation, facilitating bacterial survival in the highly acidic environment (da Cruz et al., 2009). However, no reports have described the beneficial effects of cream cheese in ALD.

Lactococcus chungangensis was isolated from sediment environments as the sixth member of the Lactococcus genus (Cho et al., 2008). This strain has been shown to 
effectively reduce both alcohol and acetaldehyde levels in short-term alcohol consumption in mice (Konkit et al., 2015a, 2016). Kimchi is a Korean traditional fermented vegetable food, known for its beneficial effects on human health (Park et al., 2014). In this study, the mitigating effects of $L$. chungangensis CAU 1447 (CAU 1447), isolated from kimchi, and cream cheese derived from CAU 1447 were evaluated to elucidate the mechanisms of alcohol-induced liver injury in a rat model of ALD.

\section{MATERIALS AND METHODS}

\section{Isolation of CAU 1447 and Culture Conditions}

Kimchi samples were obtained from Manri traditional markets in Seoul, Republic of Korea. The samples were serially diluted with PBS, spread on M17 (BD BBL, Sparks, MD) agar plates, and incubated at $30^{\circ} \mathrm{C}$ for 2 to 3 d (Terzaghi and Sandine, 1975). Cream-colored colonies were selected and identified by $16 \mathrm{rRNA}$ gene sequence analysis (Cho et al., 2008). The obtained sequences were analyzed using the NCBI BLAST program (https://blast.ncbi.nlm.nih.gov/Blast.cgi). The biochemical characteristics were determined using analytical profile index (API) 20E, API 20NE, API $50 \mathrm{CH}$, and API ZYM according to the manufacturer's instructions (bioMérieux, Marcy-l'Étoile, France). Lactococcus chungangensis CAU 1447 was one of the isolates and was used for evaluation in ALD studies. We cultured CAU 1447 on tryptic soy broth (Difco, Becton Dickinson and Company, Franklin Lakes, NJ) at $30^{\circ} \mathrm{C}$ for 2 to $3 \mathrm{~d}$, freeze-dried it, and resuspended it at a concentration of $1 \times 10^{9} \mathrm{cfu} / \mathrm{mL}$ in PBS to prepare a single dose.

\section{Ethics and Animals}

Five-week-old male Sprague-Dawley rats $(\mathrm{n}=30)$ were purchased from Central Lab Animal Inc. (Seoul, Korea) and acclimated for $1 \mathrm{wk}$ before the start of the experiment. Rats were divided into groups of 6 , and each group was separated into 2 cages with 3 rats per cage. Rats had free access to a nutritionally complete diet and water under a 12-h light-dark cycle. Temperature and humidity were maintained at $24^{\circ} \mathrm{C} \pm$ $2^{\circ} \mathrm{C}$ and $55 \pm 10 \%$, respectively. All experiments were performed according to the guidelines of the Korean Food and Drug Administration. Samples were collected from rats according to ethical guidelines mandated by the protocols presented to and approved by the ChungAng University Institutional Animal Care and Use Committee of the Laboratory Animal Research Center (approval no. 2018-00022).

\section{Ethanol Feeding}

During the experiment, rats were initially administered $50 \%$ alcohol at $4 \mathrm{~g} / \mathrm{kg} \mathrm{BW}$ daily. The dose of alcohol was then increased by $1 \mathrm{~g} / \mathrm{kg}$ every month, and the administration was continued for 2 mo. After reaching $6 \mathrm{~g} / \mathrm{kg}$ per day, administration was performed for an additional 2 wk. Alcohol was administered by oral gavage once a day.

\section{Experimental Design}

Five-week-old male Sprague-Dawley rats $(\mathrm{n}=30)$ were randomly divided into 5 groups $(\mathrm{n}=6$ /group) as follows: (1) negative control group (PBS), (2) positive control group (50\% alcohol), (3) drug control group (alcohol + silymarin), (4) CAU 1447 (alcohol + L. chungangensis CAU 1447), and (5) CAU 1447 cream cheese group (alcohol + cream cheese made with L. chungangensis CAU 1447; Supplemental Figure S1, https://doi .org/10.3168/jds.2019-16891). During the study period, silymarin, CAU 1447, and cream cheese CAU 1447 were administered $2 \mathrm{~h}$ after alcohol administration, and BW was checked daily. Silymarin (Sigma-Aldrich, St. Louis, MO) is commonly used to treat liver disease and was used in this study to compare the effects of CAU 1447 and cream cheese made with CAU 1447 on ALD. Silymarin (150 mg/kg BW daily) was dissolved in 1 mL PBS and administered by oral gavage. Freeze-dried CAU $1447\left(1 \times 10^{9} \mathrm{cfu} /\right.$ rat per day $)$ and cream cheese made with CAU 1447 (1.4 g/kg BW per rat) were dissolved in $1 \mathrm{~mL}$ PBS and administered by oral gavage. The negative control group was treated with an equal volume of PBS. All treatments lasted $10 \mathrm{wk}$. Before oral administration of alcohol, rats were pretreated with silymarin, CAU 1447, and cream cheese made with CAU 1447 for 1 week, after the acclimation period. After 10 wk of alcohol administration, rats were fasted for approximately $12 \mathrm{~h}$ before sacrificing and anesthetized by $\mathrm{CO}_{2}$ inhalation. Organs were then dissected for further analysis.

\section{Biochemical Analysis of Serum}

Before dissecting organs, anesthesia was performed with $\mathrm{CO}_{2}$, and cardiac blood was collected in $1.5-\mathrm{mL}$ microcentrifuge tubes for biochemical analysis of serum and stored at $4^{\circ} \mathrm{C}$ for approximately $1 \mathrm{~h}$. Subsequently, blood samples were centrifuged for $30 \mathrm{~min}$ at $2000 \times g$ and $4^{\circ} \mathrm{C}$ for separation of serum from whole blood. Serum was stored at $-80^{\circ} \mathrm{C}$ until biochemical analysis of alkaline phosphatase (ALP), AST, ALT, bilirubin, albumin, triglyceride, and cholesterol levels. Biochemical analysis was performed by GC Labs (Yongin, Korea) 
Analysis of Levels of Cytokines, Inflammatory Factors, and SOD from Liver

Analysis of mRNA expression levels of cytokines [tumor necrosis factor-alpha (TNFA), IFNG, IL1B, and IL10], inflammatory factors ( ${ }^{N} O S, N F K B, C O X 2$, and TLR 4), and SOD was performed using fresh and frozen liver tissues. Levels of mRNA were quantified by real-time PCR analysis. Approximately 20 to 30 $\mathrm{mg}$ of liver tissue was homogenized. For the isolation of total RNA from liver tissue, an RNeasy mini kit (Qiagen, Hilden, Germany) was used in accordance with the manufacturer's instructions. Subsequently, $50 \mu \mathrm{L}$ RNase-free water was added to the extracted RNA, and samples were stored at $-20^{\circ} \mathrm{C}$. The concentration of the extracted RNA was measured using a NanoQuant spectrophotometer (Infinite 200; Tecan, Männedorf, Switzerland). Next, $1 \mu \mathrm{g}$ total RNA was reverse transcribed into cDNA using a PrimeScript first-strand cDNA synthesis kit (Takara Bio, Shiga, Japan). Quantification of mRNA expression was then performed using real-time quantitative $\mathrm{PCR}$ with a 7500 Fast real-time PCR system (Applied Biosystems, Foster City, CA) and a SYBR Green PCR kit (Qiagen). Fold changes were determined using the $2^{-\Delta \Delta \mathrm{Ct}}$ method. The primer sequences used are shown in Supplemental Tables S1 and S2 (https://doi.org/10.3168/jds.2019 -16891). Glyceraldehyde 3-phosphate dehydrogenase $(G A P D H)$ was used as a control.

\section{Liver Antioxidant Enzyme Activity Analysis}

Liver antioxidant enzymes and SOD activity were measured using a Superoxide Dismutase Assay kit (Cayman Chemical, Ann Arbor, MI) in accordance with the manufacturer's method. Small sections of rat liver tissues were stored in liquid nitrogen until analysis. Liver tissues were homogenized in cold HEPES buffer containing $210 \mathrm{~m} M$ mannitol, $1 \mathrm{~m} M$ EGTA, and 70 $\mathrm{m} M$ sucrose and centrifuged at $1,500 \times g$ for $5 \mathrm{~min}$ at $4^{\circ} \mathrm{C}$. Aliquots of supernatant were transferred to sterilized microcentrifuge tubes and stored at $-80^{\circ} \mathrm{C}$ until antioxidant enzyme activity analysis. The activity was determined as units per milliliter. A microplate reader was used to measure the absorbance at $450 \mathrm{~nm}$.

\section{Short-Chain Fatty Acids in Fecal Samples}

Fecal samples were collected every week, and the concentrations of short-chain fatty acids (SCFA) were determined using HPLC (Ultimate 3000; Thermo Scientific Dionex, Sunnyvale, CA). Next, 4-methylvaleric acid $(0.25 \mathrm{~m} M)$ was added to $100-\mathrm{mg}$ fecal samples, and the samples were vortexed and centrifuged at 12,000 $\times$ $g$ for $5 \mathrm{~min}$. The supernatants were discarded, and the pellets were mixed with distilled water, vortexed, and centrifuged at $12,000 \times g$ for $5 \mathrm{~min}$. The supernatants were then filtered, and aliquots of supernatants were transferred to disposable centrifuge tubes and stored at $-20^{\circ} \mathrm{C}$ until required for HPLC analysis. Butyrate and acetate acid were analyzed by HPLC. The composition of each SCFA was presented as a percentage of total SCFA (mg/L).

\section{Histological Analysis}

Fresh liver and intestinal tissues were carefully separated, cut into 3- to 5- $\mu$ m-thick sections, fixed in $10 \%$ formalin, and embedded in paraffin. After $24 \mathrm{~h}$, paraffin-embedded tissues were stained with hematoxylin and eosin. Tissue samples were then observed by microscopy (DM 4000B; Leica Microsystems, Wetzlar, Germany) at $400 \times$ magnification for assessment of histopathological changes. At least 3 slides were evaluated for each sample.

\section{Statistical Analysis}

Statistical data were analyzed using GraphPad Prism (v. 7.0, GraphPad Software Inc., La Jolla, CA), and data are displayed as means \pm standard errors of the means. To compare the effects of more than 2 groups, one-way ANOVA was used with Bonferroni's post-hoc tests. Results with $P$-values of less than 0.05 were considered significant.

\section{RESULTS}

\section{Liver-to-BW Ratios}

We provided diets in fixed calories of $56 \mathrm{kcal} /$ pellet per rat daily, and dietary intake was not significantly different among the experimental groups. We measured BW and liver weights to determine the liver-to-BW ratios. Notably, liver weights increased in the alcoholtreated positive control group (Supplemental Figure S2A, https://doi.org/10.3168/jds.2019-16891), and the liver/BW ratio was highest in the alcohol-treated positive control group (Supplemental Figure S2B, https:/ /doi.org/10.3168/jds.2019-16891). In contrast, we observed decreased liver/BW ratios in the CAU 1447 and CAU 1447 cream cheese groups, resulting in liver/BW ratios similar to those in the negative control group. 

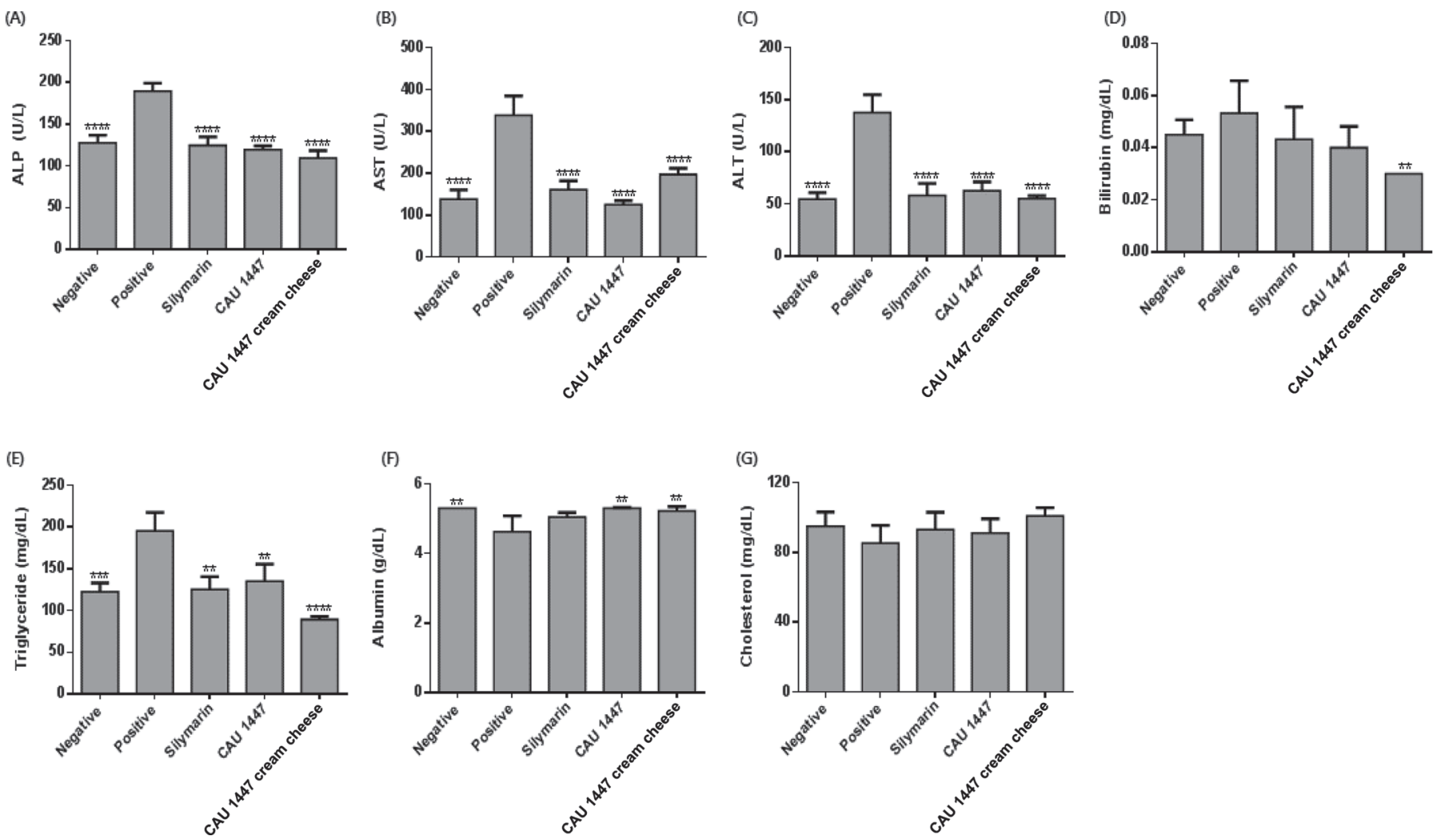

Figure 1. Effects of treatment with Lactococcus chungangensis CAU 1447 (CAU 1447) and CAU 1447 cream cheese on biochemical parameters of liver function in rat serum: (A) alkaline phosphatase (ALP), (B) aspartate aminotransferase (AST), (C) alanine aminotransferase (ALT), (D) bilirubin, (E) triglycerides, $(\mathrm{F})$ albumin, and $(\mathrm{G})$ cholesterol. Data are presented as means, and error bars indicate $\mathrm{SE}$. ${ }^{* *} P<0.005 ; * * * P$ $<0.0005 ;{ }^{* * * *} P<0.0001$, compared with the positive control group.

\section{Biochemical Parameters Associated with Liver Function}

Next, we evaluated changes in biochemical factors related to liver injury and fatty liver using serum samples. We observed significant increases in serum levels of ALP, AST, and ALT in the alcohol-treated positive control group. However, we observed reduced serum levels of ALP, AST, and ALT in the silymarin-treated, CAU 1447, and CAU 1447 cream cheese groups compared with those in the positive control group (Figure $1 \mathrm{~A}-1 \mathrm{C})$. Moreover, in the experimental groups, serum levels of ALP, AST, and ALT were similar to those in the negative control group. In the CAU 1447 and CAU 1447 cream cheese groups, serum levels of ALP, AST, and ALT were higher than those in the silymarin group but similar to those in the negative control group. Moreover, bilirubin and triglyceride serum levels were lower in the silymarin, CAU 1447, and CAU 1447 cream cheese groups than in the positive control group (Figure 1D and 1E). In contrast, serum albumin and cholesterol levels were lowest in the positive control group (Figure $1 \mathrm{~F}$ and $1 \mathrm{G}$ ); however, cholesterol levels did not differ among the groups.

\section{mRNA Expression Levels}

Interestingly, the expression levels of inflammatory cytokines, including TNFA, IFNG, IL1B, and IL10, increased significantly in liver tissues from rats in the alcohol-treated positive control group (Figure 2). Oral administration of CAU 1447 and CAU 1447 cream cheese suppressed the induction of these cytokines. Moreover, the expression levels of these cytokines were similar to or lower than those in the negative control group. In the drug-treated group, the results were similar to those in the CAU 1447 and CAU 1447 cream cheese groups.

\section{Expression of NF-KB, COX-2, iNOS, TLR4, and SOD}

Evaluation of the mRNA expression levels of inflammatory factors and antioxidant enzymes showed NFKB, COX2, iNOS, TLR 4, and SOD mRNA upregu- 
lated levels in the alcohol-treated positive control group compared with those in the normal group (Figure 3). However, treatment with CAU 1447 and CAU 1447 cream cheese after alcohol administration blocked these increases. In addition, we observed similar decreases in NFKB, COX2, iNOS, TLR4, and SOD levels in the silymarin-treated drug group.

\section{Liver Antioxidant Enzyme Activity}

We next evaluated the activity of SOD, a liver antioxidant enzyme that is expressed in the fatty liver and during liver injury and is induced by high concentrations of alcohol. Compared with the untreated negative control group, we observed significantly increased SOD activities in the alcohol-treated positive control group (Figure 4). However, groups treated with CAU 1447
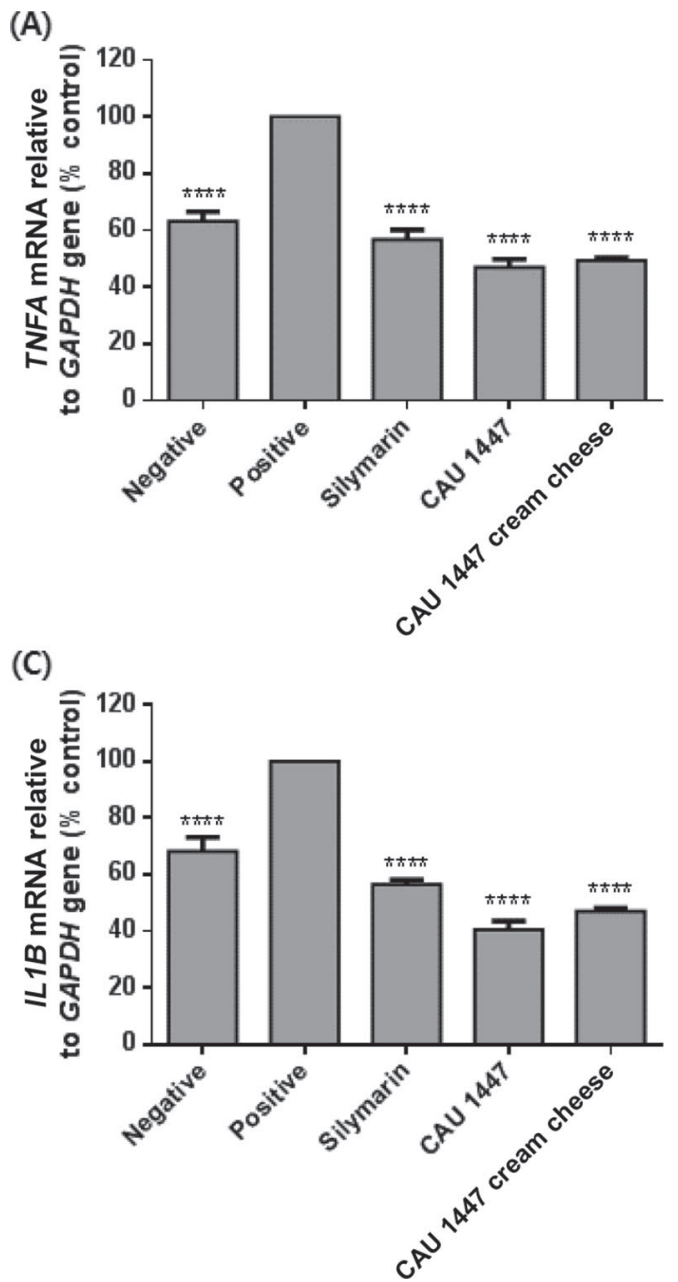

and CAU 1447 cream cheese showed decreased SOD activities compared with those in the positive control group, showing activity levels similar to those in the negative control group. In the silymarin-treated drug group, the SOD level significantly decreased, resulting in low antioxidant activity.

\section{Gut SCFA Levels}

We next evaluated SCFA production by gut microbiota, via fecal samples. Notably, butyrate and acetate levels decreased in the positive control group compared with those in the negative control group (Figure 5A and 5B). Moreover, fecal samples in the CAU 1447 and CAU 1447 cream cheese groups had similar or higher levels of butyrate and acetate than did the positive control group, whereas levels of butyrate and acetate
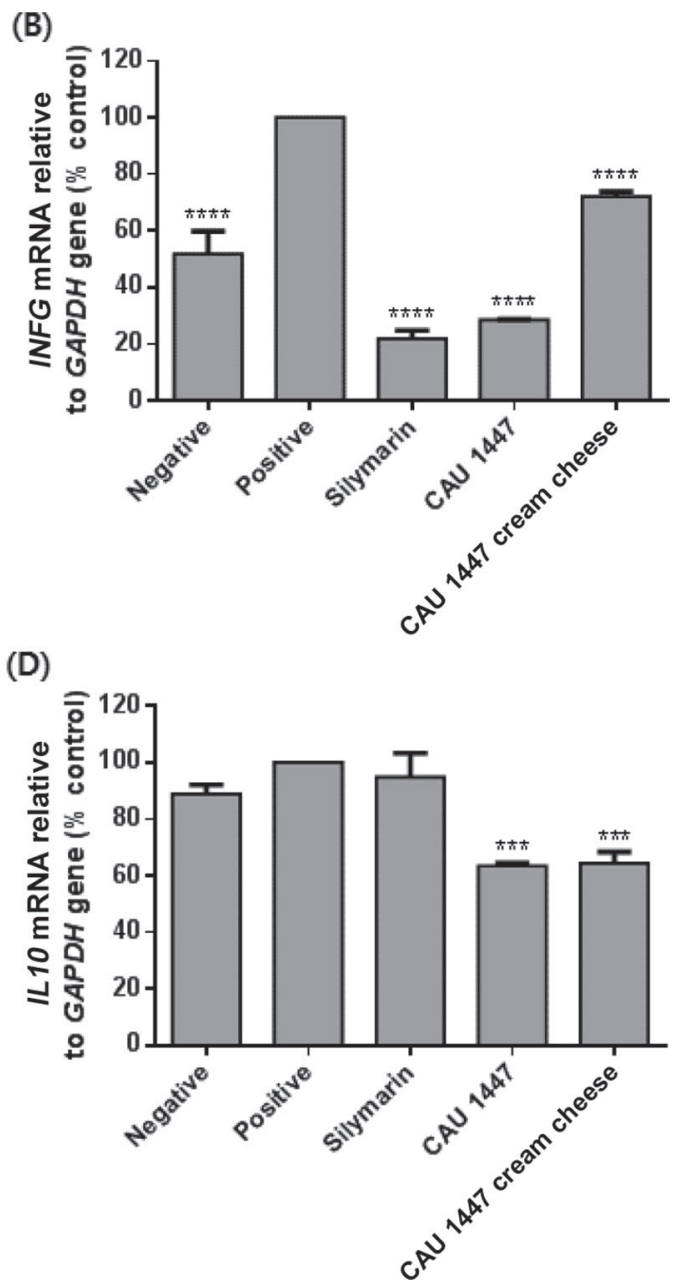

Figure 2. Effects of treatment with Lactococcus chungangensis CAU 1447 (CAU 1447) and CAU 1447 cream cheese on mRNA expression of cytokines in rat liver tissues: (A) tumor necrosis factor- $\alpha$ (TNFA), (B) IFNG, (C) IL1B, and (D) IL10 were quantified by real-time PCR. Data are presented as means, and error bars indicate SE. ${ }^{* * *} P<0.0005 ; * * * * P 0.0001$, compared with the positive control group $(G A P D H)$. 
(A)
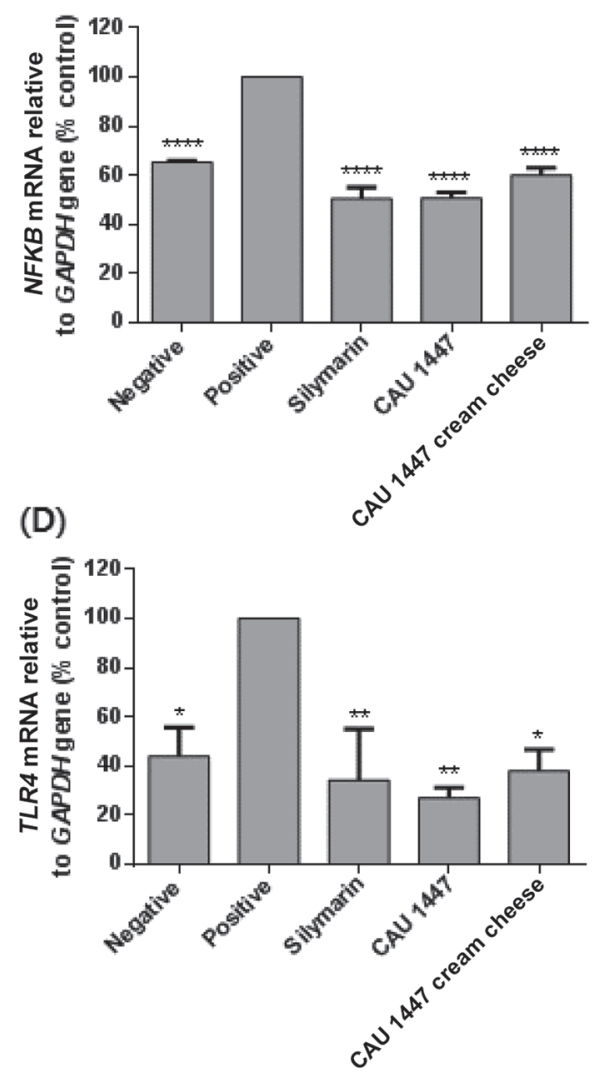

(B)
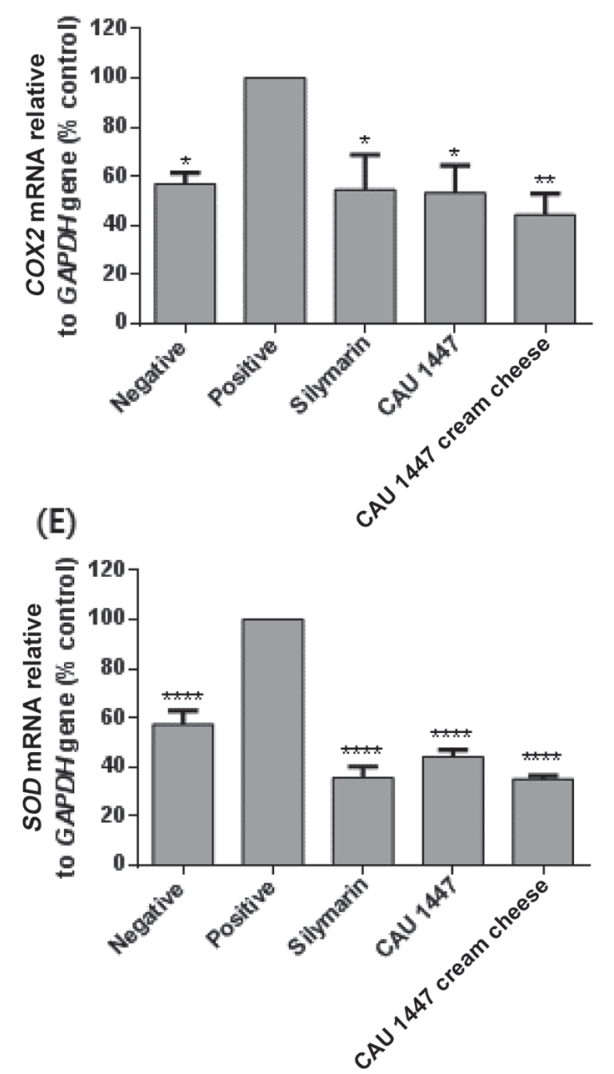

(C)

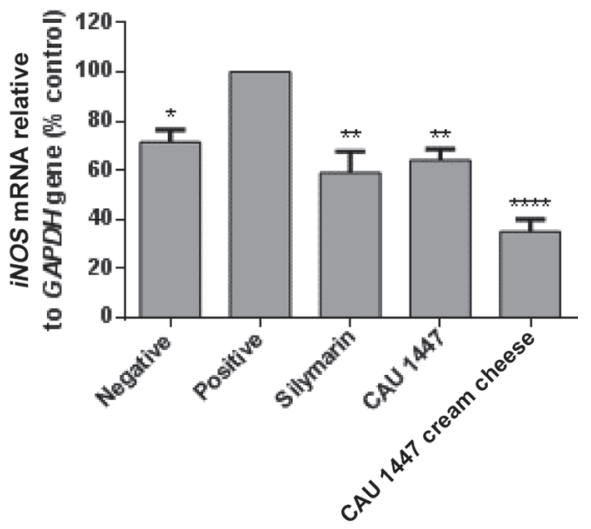

Figure 3. Expression of (A) nuclear factor- $\mathrm{B}(N F K B)$, (B) cyclooxygenase-2 (COX2), (C) inducible nitric oxide synthase (iNOS), (D) tolllike receptor-4 (TLR4), and (E) superoxide dismutase $(S O D)$ in liver samples from rats treated with Lactococcus chungangensis CAU 1447 (CAU $1447)$ and CAU 1447 cream cheese. Levels were quantified by real-time PCR. Data are presented as means, and error bars indicate SE. ${ }^{*} P<$ $0.05 ;{ }^{* *} P<0.005 ;{ }^{* * * *} P<0.0001$, compared with the positive control group $(G A P D H)$.

slightly increased in the CAU 1447 cream cheese group compared with those in the CAU 1447 group. Additionally, the acetate/butyrate ratio decreased in the CAU 1447 cream cheese group compared with those in the positive control and drug-treated groups (Figure $5 \mathrm{C}$ ).

\section{Histopathological Changes}

We also examined liver cell structure, lobular architecture, and intestinal structure via staining with hematoxylin and eosin. In the negative control group, we did not observe liver injury or histopathological changes (Figure 6A). In the alcohol-treated positive control group (Figure 6B), hepatocytes surrounding the central vein of the liver revealed injury and contained lipid droplets. In the drug-treated group (Figure 6C), results were similar to those in the CAU 1447 and CAU 1447 cream cheese groups. However, oral administration of silymarin, CAU 1447, or CAU 1447 cream cheese decreased these phenotypes and prevented occurrence of injury and formation of lipid droplets (Figure 6D and
$6 \mathrm{E})$. The structure of the intestinal villi in the negative control group showed no morphological changes (Figure 7A). In contrast, in the alcohol-treated positive control group (Figure 7B), we observed changes in the intestinal villus structure; intestinal villi were greatly damaged and ripped. In the drug-treated group, the results were similar to those in the CAU 1447 and CAU 1447 cream cheese groups (Figure 7C). However, oral administration of CAU 1447 or CAU 1447 cream cheese (Figure 7D and 7E) significantly decreased injury to the intestinal villi compared with the positive control group.

\section{DISCUSSION}

Excessive, long-term alcohol absorption causes ALD and liver injury. Results of the current study showed that alcohol treatment increased ALP, AST, and ALT levels in serum, suggesting that alcohol absorption could affect hepatitis, fatty liver, and intestinal injury; AST and ALT are markers of hepatocytes and are released 
from the liver following hepatocyte damage (Sass and Shaikh, 2006). However, supplementation with CAU 1447 or CAU 1447 cream cheese significantly prevented the increases in serum ALP, AST, and ALT levels caused by alcohol consumption. These results are supported by several previous studies demonstrating that treatment with some probiotics containing Lactobacillus spp., such as Lactobacillus brevis and Lactobacillus casei, and Bifidobacteria suppressed the upregulation of biomarkers or inflammatory factors related to alcoholic liver injury in patients and animal models (Segawa et al., 2008; Stadlbauer et al., 2008; Tian et al., 2017). Therefore, our results demonstrated decreases in ALP, AST, and ALT levels following supplementation with CAU 1447 or CAU 1447 cream cheese.

In this study, treatment with CAU 1447 and CAU 1447 cream cheese significantly reduced the levels of inflammatory factors and cytokines, such as NF- $\kappa B$, COX-2, iNOS, TLR4, SOD, TNF- $\alpha$, IFN $\gamma$, IL-1 $\beta$, and IL-10, in liver tissue. The pathogenesis of liver dam-

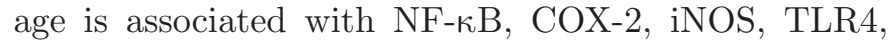
and SOD. Activity of NF- $\kappa \mathrm{B}$ and TLR4 expression are involved in inflammatory responses and regulate the levels of inflammatory cytokines. Additionally, TLR4 is related to liver disease and has been shown to be associated with inflammation, necrosis, and steatosis

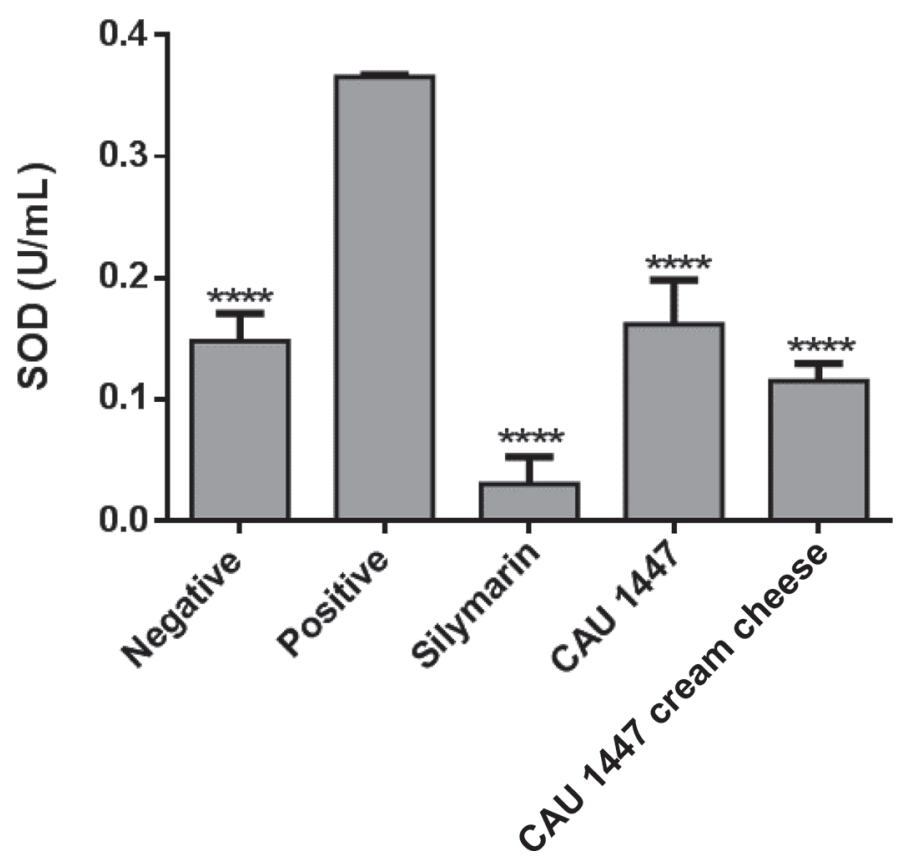

Figure 4. Effects of treatment with Lactococcus chungangensis CAU 1447 (CAU 1447) and CAU 1447 cream cheese on antioxidant activity in rat liver tissues. Superoxide dismutase activity (SOD) was examined using a SOD assay kit. Data are presented as means, and error bars indicate SE. ${ }^{* * * *} P<0.0001$, compared with the positive control group. following alcohol absorption (Inokuchi et al., 2011). Moreover, COX-2 and iNOS expression levels are affected by $\mathrm{NF}-\kappa \mathrm{B}$ activation. In $\mathrm{ALD}$, upregulation of proinflammatory factors, such as products of lipid peroxidation, endotoxins, and cytokines, induces COX2 expression, which then enhances the inflammatory response in the liver (Lee et al., 1992; O'Sullivan et al., 1992; Feng et al., 1995).

Notably, we observed low mRNA expression levels of TNFA, IFNG, IL1B, and IL10 in the CAU 1447 and CAU 1447 cream cheese groups. Generally, TNF- $\alpha$, IL-1 $\beta$, and IL-10 expression is induced in the liver following alcohol treatment. For instance, IL-10 inhibits functions related to reactive oxygen, nitric oxide, and proinflammatory cytokine synthesis (Moore et al., 1993). Moreover, in ALD, upregulation of TNF- $\alpha$ stimulates various cytokines or chemokines to induce steatohepatitis and promote the development of liver injury (Kawaratani et al., 2013). Also thought to be involved in systemic inflammation, $\mathrm{TNF}-\alpha$ is a crucial factor involved in the progression of ALD (Kitazawa et al., 2003). A dominant proinflammatory cytokine, IL-1 $\beta$ is upregulated in the liver in animal models of ALD and in patients with ALD (Petrasek et al., 2012). Together, the expression of these proinflammatory cytokines results in hepatocyte malfunction, inflammation, necrosis, and apoptosis in liver injury (Tilg et al., 2016). Therefore, the present results demonstrated that administration of CAU 1447 and CAU 1447 cream cheese may prevent liver injury.

The activity of antioxidant enzymes released from the liver, such as SOD, is another indicator of liver injury. In this study, the activity of SOD was increased by alcohol treatment but decreased in the CAU 1447 and CAU 1447 cream cheese groups. The enzyme SOD converts the superoxide anion to hydrogen peroxide and acts to detoxify toxins, such as free radicals, generated in the body. The increased activity of SOD suggested that reactive oxygen species generated by alcohol-induced liver injury were eliminated (Whang et al., 1999).

It is commonly known that alcohol induces alterations of gut flora, increases gut permeability, and causes mucosal damage, leading to transposition of endotoxins and bacterial substances via inflammation induction (Vassallo et al., 2015). However, a study demonstrated that Lactobacillus rhamnosus GG has protective effects on the intestinal mucus layer (Wang et al., 2012).

Short-chain fatty acids play important roles in human health, including in immune responses and in the inflammatory system. Moreover, the composition of SCFA differs according to interactions with the gut microbiota and gut environment (Morrison and Preston, 2016). A previous study using L. chungangensis CAU 28 and L. chungangensis CAU 28 cream cheese sug- 
(A)

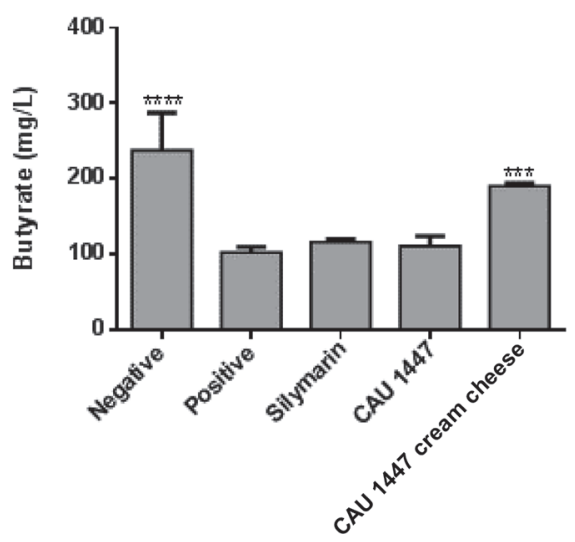

(B)

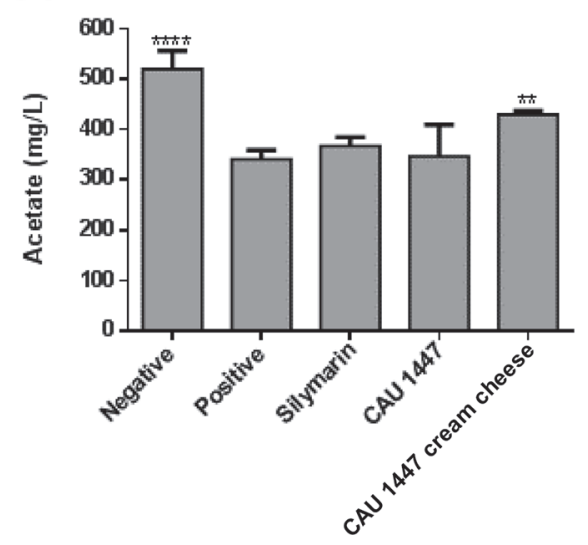

(C)

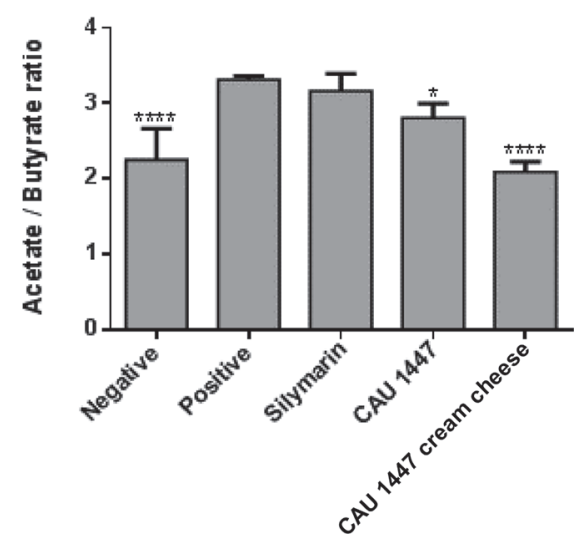

Figure 5. Effects of treatment with Lactococcus chungangensis CAU 1447 (CAU 1447) and CAU 1447 cream cheese on short-chain fatty acid (SCFA) levels. (A) Butyrate, (B) acetate, and (C) the ratio of acetate to butyrate were evaluated by HPLC using fecal samples. Data are presented as means, and error bars indicate SE. ${ }^{*} P<0.05 ;{ }^{* *} P<0.005$; ${ }^{* * *} P<0.0005 ;{ }^{* * * *} P<0.0001$, compared with the positive control group.

(A)

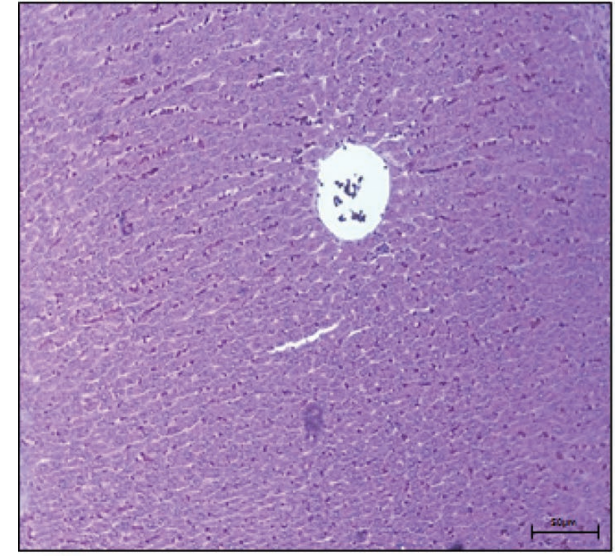

(C)

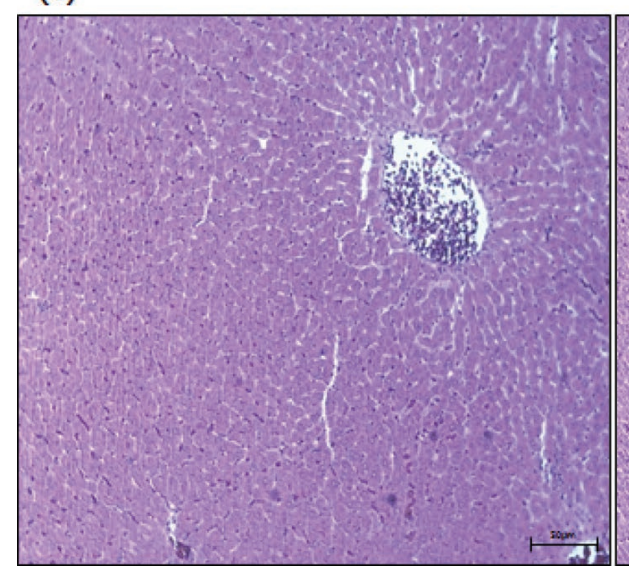

(B)

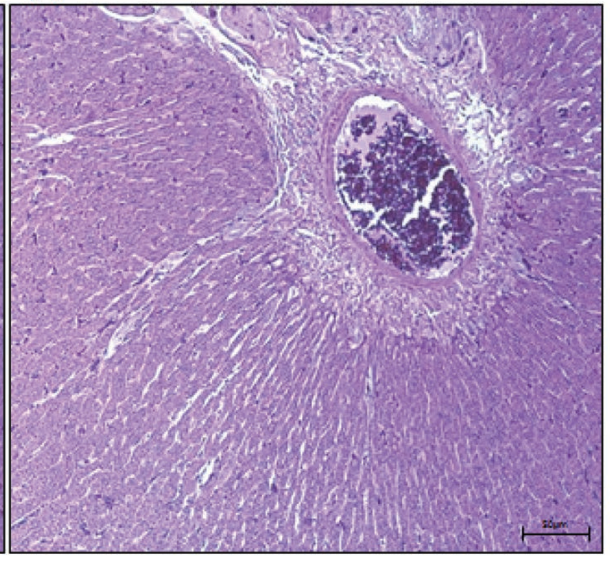

(D)

(E)

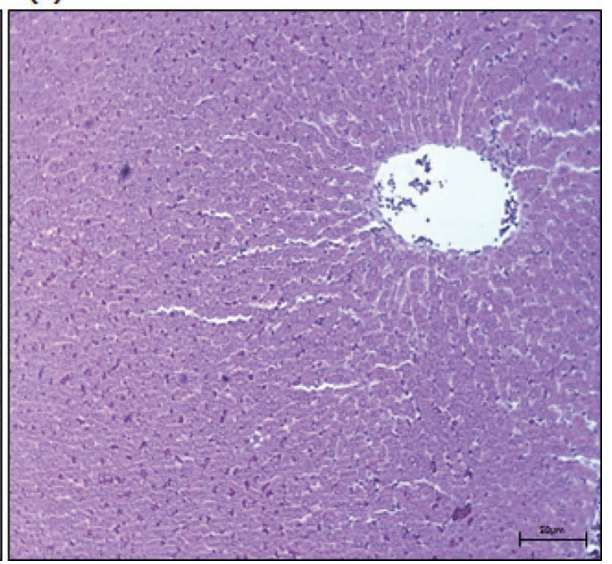

Figure 6. Effects of treatment with Lactococcus chungangensis CAU 1447 (CAU 1447) and CAU 1447 cream cheese on liver histology. The structure of the liver in the (A) negative control group, (B) positive control group, (C) drug-treated group, (D) CAU 1447 group, and (E) CAU 1447 cream cheese group, as determined by staining with hematoxylin and eosin. Scale bars $=50 \mu \mathrm{m}$. 
(A)

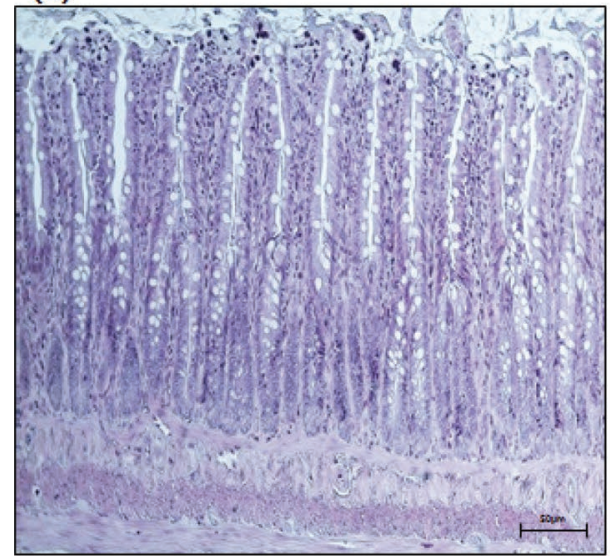

(C)

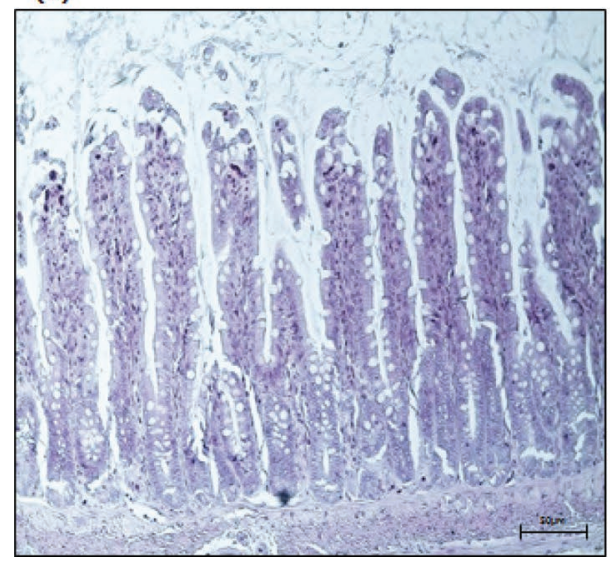

(B)

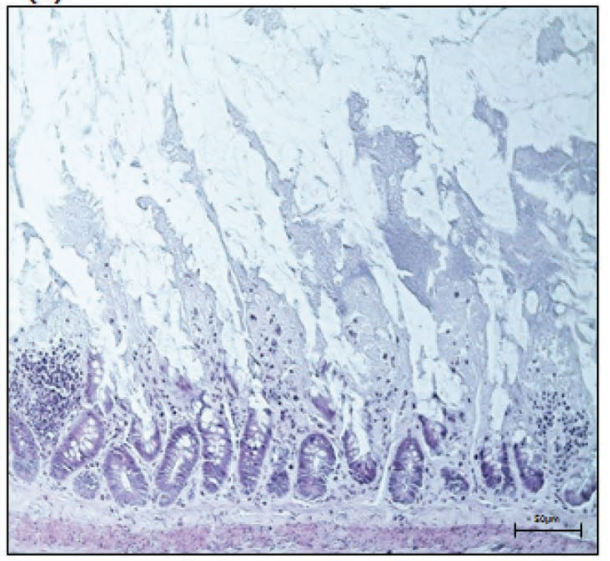

(D)

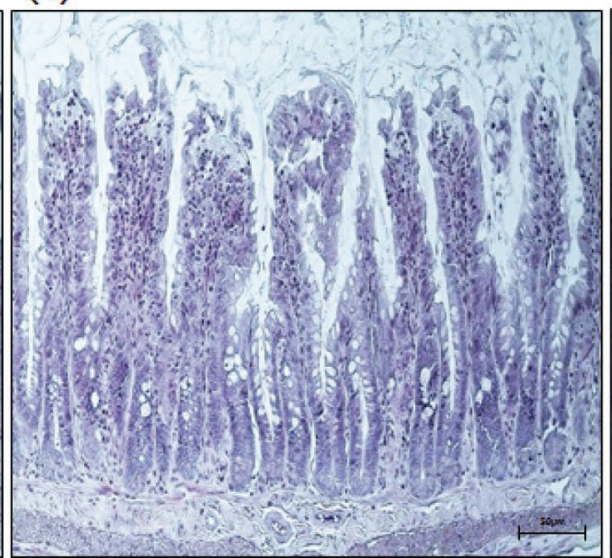

(E)

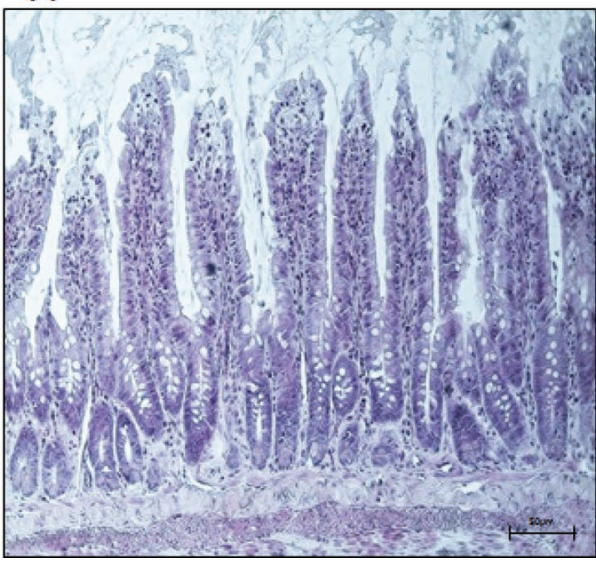

Figure 7. Effects of treatment with Lactococcus chungangensis CAU 1447 (CAU 1447) and CAU 1447 cream cheese on intestinal histology. Intestinal structures in the (A) negative control group, (B) positive control group, (C) drug-treated group, (D) CAU 1447 group, and (E) CAU 1447 cream cheese group, as determined by staining with hematoxylin and eosin. Scale bars $=50 \mu \mathrm{m}$.

gested the improvement of gut homeostasis and health through the elevation of SCFA production (Kim et al., 2019). Further, another study showed that high levels of butyrate are related to regulation of the immune response, including inhibition of $\mathrm{TNF}-\alpha$ by inactivation

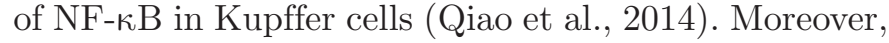
butyrate suppresses the expression of lipopolysaccharide-induced cytokines (Nastasi et al., 2015). The SCFA stimulate inflammatory responses by association with the host and microbiome or by directly affecting signaling. The liver contains various cell types that interact with small molecules produced by the gut microbiota to facilitate immune signaling (Morrison and Preston, 2016). In the current study, the CAU 1447 cream cheese group showed an elevation of butyrate and acetate levels in fecal samples compared with those in the positive control group. Additionally, oral administration of CAU 1447 and CAU 1447 cream cheese decreased the ratio of acetate to butyrate compared with that of the positive control group. Colonic morphology is known to be associated with a higher acetate-to-butyrate ratio (Shah et al., 2018). Taken together, these results suggested that high levels of butyrate and acetate increase the metabolic activity in the gut and accelerate the immune response.

Oral administration of $L$. chungangensis CAU 1447 and cream cheese derived therefrom effectively prevented alcohol-induced liver injury, as indicated by downregulation of AST, ALT, ALP, inflammatory factors, and cytokines. Moreover, treatment with CAU 1447 cream cheese also produced higher levels of SCFA, to prevent ALD (Figure 8). These results suggested that L. chungangensis CAU 1447 may have applications as a supplement for alleviation of ALD. Further studies and clinical trials are needed to confirm these findings.

\section{ACKNOWLEDGMENTS}

This work was supported in part by grant 20180631 from Medikan Co. Ltd. (Seoul, Korea). 


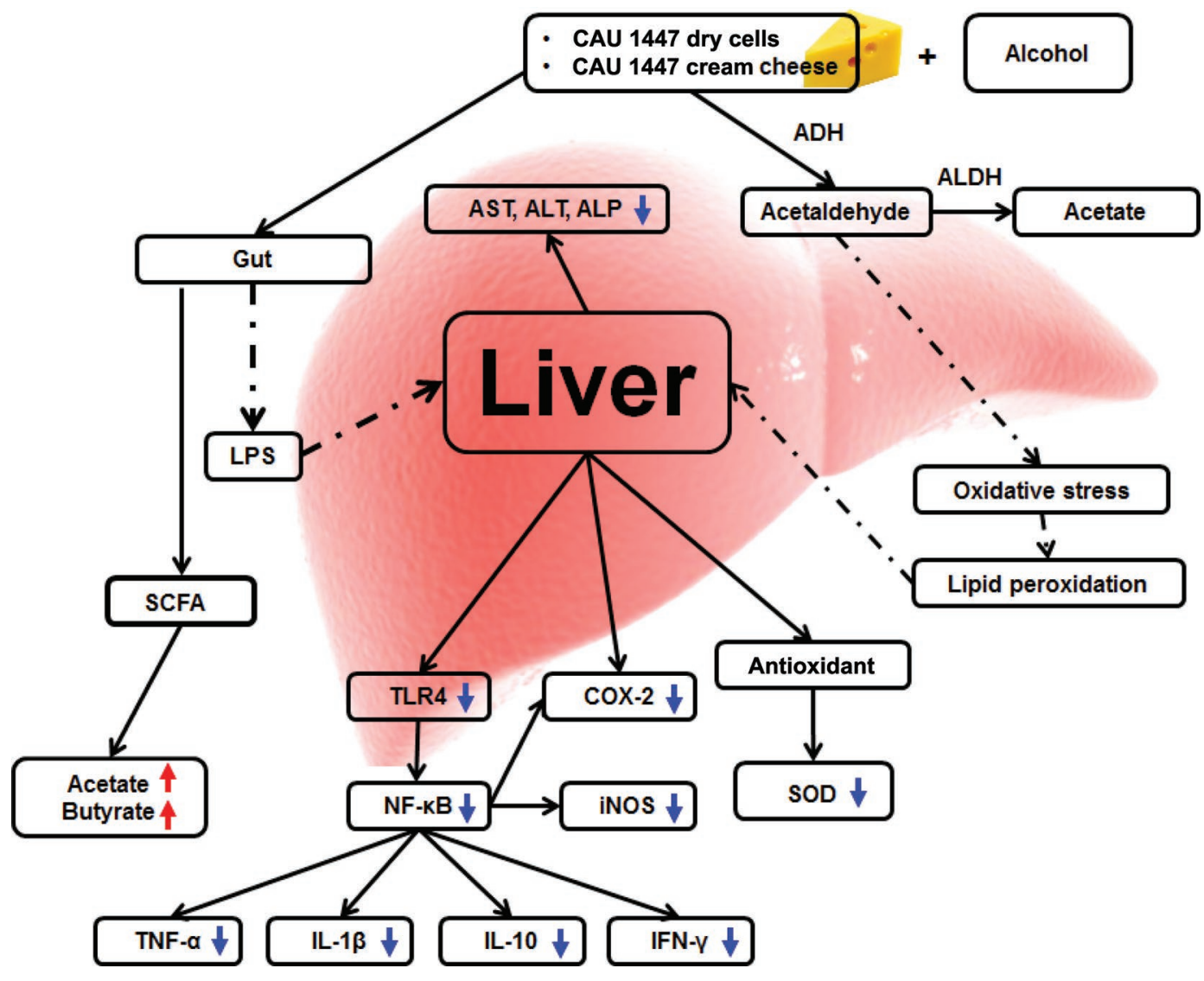

Figure 8. Overview of the mechanisms mediating the effects of treatment with Lactococcus chungangensis CAU 1447 cream cheese on alcohol absorption. A brief schematic of the study results and expected mechanisms demonstrating the roles of inflammatory mediators, immune responses, antioxidant activities, and SCFA in alcoholic liver disease. The mechanistic links are represented by solid lines (direct links) and dotted lines (speculative links). AST = aspartate aminotransferase; ALT = alanine aminotransferase; ALP = alkaline phosphatase; ADH $=$ alcohol dehydrogenase; $\mathrm{ALDH}=$ aldehyde dehydrogenase; SCFA = short-chain fatty acids; TLR4 = toll-like receptor-4; COX-2 = cyclooxygenase-2; $\mathrm{NF}-\kappa \mathrm{B}=$ nuclear factor- $\kappa \mathrm{B}$; iNOS $=$ inducible nitric oxide synthase; SOD = superoxide dismutase; TNF- $\alpha=$ tumor necrosis factor- $\alpha$.

\section{REFERENCES}

Barone, R., F. Rappa, F. Macaluso, C. C. Bavisotto, C. Sangiorgi, G. D. Paola, G. Tomasello, V. D. Felice, V. Marciano, F. Farina, G. Zummo, E. C. Macario, A. J. L. Macario, M. Cocchi, F. Cappello, and A. M. Gammazza. 2016. Alcoholic liver disease: A mouse model reveals protection by Lactobacillus fermentum. Clin. Transl. Gastroenterol. 7:e138.

Cho, S. L., S. W. Nam, J. H. Yoon, J. S. Lee, A. Sukhoom, and W. Kim. 2008. Lactococcus chungangensis sp. nov., a lactic acid bacterium isolated from activated sludge foam. Int. J. Syst. Evol. Microbiol. 58:1844-1849.

Chuang, C. H., C. C. Tsai, E. S. Lin, C. S. Huang, Y. Y. Lin, C. C Lan, and C. C. Huang. 2016. Heat-killed Lactobacillus salivarius and Lactobacillus johnsonii reduce liver injury induced by alcohol in vitro and in vivo. Molecules 21:E1456.

da Cruz, A. G., F. C. A. Buriti, C. H. B. de Souza, J. A. F. Faria, and S. M. I. Saad. 2009. Probiotic cheese: Health benefits, technological and stability aspects. Trends Food Sci. Technol. 20:344-354.

Feng, L., Y. Xia, G. E. Garcia, D. Hwang, and C. B. Wilson. 1995. Involvement of reactive oxygen intermediates in cyclooxygenase-2 expression induced by interleukin-1, tumor necrosis factor-alpha, and lipopolysaccharide. J. Clin. Invest. 95:1669-1675.

Gyamfi, M. A., and Y. J. Y. Wan. 2010. Pathogenesis of alcoholic liver disease: The role of nuclear receptors. Exp. Biol. Med. (Maywood) 235:547-560
Inokuchi, S., H. Tsukamoto, E. Park, Z. X. Liu, D. A. Brenner, and E. Seki. 2011. Toll-like receptor 4 mediates alcohol-induced steatohepatitis through bone marrow-derived and endogenous liver cells in mice. Alcohol. Clin. Exp. Res. 35:1509-1518.

Kawaratani, H., T. Tsujimoto, A. Douhara, H. Takaya, K. Moriya, T. Namisaki, R. Noguchi, H. Yoshiji, M. Fujimoto, and H. Fukui. 2013. The effect of inflammatory cytokines in alcoholic liver disease. Mediators Inflamm. 2013:495156.

Kim, J. H., K. Kim, and W. Kim. 2019. Cream cheese-derived Lactococcus chungangensis CAU 28 modulates the gut microbiota and alleviates atopic dermatitis in BALB/c mice. Sci. Rep. 9:446.

Kitazawa, T., Y. Nakatani, M. Fujimoto, N. Tamura, M. Uemura, and H. Fukui. 2003. The production of tumor necrosis factor-alpha by macrophages in rats with acute alcohol loading. Alcohol. Clin. Exp. Res. 27:72S-75S.

Konkit, M., W. J. Choi, and W. Kim. 2015. Alcohol dehydrogenase activity in Lactococcus chungangensis: Application in cream cheese to moderate alcohol uptake. J. Dairy Sci. 98:5974-5982.

Konkit, M., W. J. Choi, and W. Kim. 2016. Aldehyde dehydrogenase activity in Lactococcus chungangensis: Application in cream cheese to reduce aldehyde in alcohol metabolism. J. Dairy Sci. 99:1755-1761.

Lee, S. H., E. Soyoola, P. Chanmugam, S. Hart, W. Sun, H. Zhong, S. Liou, and D. Simmons. 1992. Selective expression of mitogen-inducible cyclooxygenase in macrophages stimulated with lipopolysaccharide. J. Biol. Chem. 267:25934-25938. 
Liu, H., X. Qi, S. Cao, and P. Li. 2014. Protective effect of flavonoid extract from Chinese bayberry (Myrica rubra Sieb. et Zucc.) fruit on alcoholic liver oxidative injury in mice. J. Nat. Med. 68:521-529.

Lyu, Y., L. Zhong, Y. Liu, J. Lu, G. LaPointe, F. Lu, and Z. Lu. 2018. Protective effects of Lactococcus lactis expressing alcohol dehydrogenase and acetaldehyde dehydrogenase on acute alcoholic liver injury in mice. J. Chem. Technol. Biotechnol. 93:1502-1510.

Medzhitov, R., P. Preston-Hurlburt, and C. A. Janeway Jr.. 1997. A human homologue of the Drosophila Toll protein signals activation of adaptive immunity. Nature 388:394-397.

Moore, K. W., A. O'Garra, R. D. W. Malefyt, P. Vieira, and T. R. Mosmann. 1993. Interleukin-10. Annu. Rev. Immunol. 11:165-190.

Morrison, D. J., and T. Preston. 2016. Formation of short chain fatty acids by the gut microbiota and their impact on human metabolism. Gut Microbes 7:189-200.

Nastasi, C., M. Candela, C. M. Bonefeld, C. Geisler, M. Hansen, T. Krejsgaard, E. Biagi, M. H. Andersen, P. Brigidi, N. Ødum, T. Litman, and A. Woetmann. 2015. The effect of short-chain fatty acids on human monocyte-derived dendritic cells. Sci. Rep. 5:16148.

O'Shea, R. S., S. Dasarathy, and A. J. McCullough. 2010. Alcoholic liver disease. Hepatology 51:307-328.

O'Sullivan, M. G., F. H. Chilton, E. M. Huggins, and C. E. McCall. 1992. Lipopolysaccharide priming of alveolar macrophages for enhanced synthesis of prostanoids involves induction of a novel prostaglandin H synthase. J. Biol. Chem. 267:14547-14550.

Park, K. Y., J. K. Jeong, Y. E. Lee, and J. W. Daily 3rd.. 2014. Health benefits of kimchi (Korean fermented vegetables) as a probiotic food. J. Med. Food 17:6-20.

Petrasek, J., S. Bala, T. Csak, D. Lippai, K. Kodys, V. Menashy, M. Barrieau, S. Y. Min, E. A. Kurt-Jones, and G. Szabo. 2012. IL-1 receptor antagonist ameliorates inflammasome-dependent alcoholic steatohepatitis in mice. J. Clin. Invest. 122:3476-3489.

Qiao, Y. L., J. M. Qian, F. R. Wang, Z. Y. Ma, and Q. W. Wang. 2014. Butyrate protects liver against ischemia reperfusion injury by inhibiting nuclear factor kappa B activation in Kupffer cells. J. Surg. Res. 187:653-659.

Rehm, J., A. V. Samokhvalov, and K. D. Shield. 2013. Global burden of alcoholic liver diseases. J. Hepatol. 59:160-168.

Ruggirello, M., M. Giordano, M. Bertolino, I. Ferrocino, L. Cocolin, and P. Dolci. 2018. Study of Lactococcus lactis during advanced ripening stages of model cheeses characterized by GC-MS. Food Microbiol. 74:132-142.

Sass, D. A., and O. S. Shaikh. 2006. Alcoholic hepatitis. Clin. Liver Dis. 10:219-237.

Segawa, S., Y. Wakita, H. Hirata, and J. Watari. 2008. Oral administration of heat-killed Lactobacillus brevis SBC8803 ameliorates alcoholic liver disease in ethanol-containing diet-fed C57BL/6N mice. Int. J. Food Microbiol. 128:371-377.

Shah, N. 2018. Effect of increased ratio of butyrate to physiological concentrations of acetate and propionate on intestinal integrity and IL-8 secretion in Caco-2 cells. MS thesis, Department of Food Science and Human Nutrition, Iowa State University, Ames.
Shukla, P. K., A. S. Meena, B. Manda, M. Gomes-Solecki, P. Dietrich, I. Dragatsis, and R. Rao. 2018. Lactobacillus plantarum prevents and mitigates alcohol-induced disruption of colonic epithelial tight junctions, endotoxemia, and liver damage by an EGF receptor-dependent mechanism. FASEB J. 32:6274-6292.

Stadlbauer, V., R. P. Mookerjee, S. Hodges, G. A. K. Wright, N. A. Davies, and R. Jalan. 2008. Effect of probiotic treatment on deranged neutrophil function and cytokine responses in patients with compensated alcoholic cirrhosis. J. Hepatol. 48:945-951.

Suetsugu, H., Y. Iimuro, T. Uehara, T. Nishio, N. Harada, M. Yoshida, E. Hatano, G. Son, J. Fujimoto, and Y. Yamaoka. 2005. Nuclear factor $\mathrm{\kappa B}$ inactivation in the rat liver ameliorates short term total warm ischaemia/reperfusion injury. Gut 54:835-842.

Suo, H., X. Zhao, Y. Qian, P. Sun, K. Zhu, J. Li, and B. Sun. 2016. Lactobacillus fermentum Suo attenuates $\mathrm{HCl}$ /ethanol induced gastric injury in mice through its antioxidant effects. Nutrients 8:155.

Terzaghi, B. E., and W. E. Sandine. 1975. Improved medium for lactic streptococci and their bacteriophages. Appl. Microbiol. 29:807813

Tian, F., L. Yu, Q. Zhai, Y. Xiao, Y. Shi, J. Jiang, X. Liu, J. Zhao, and H. Zhang. 2017. The therapeutic protection of a living and dead Lactobacillus strain against aluminum-induced brain and liver injuries in C57BL/6 mice. PLoS One 12:e0175398.

Tilg, H., A. R. Moschen, and G. Szabo. 2016. Interleukin-1 and inflammasomes in alcoholic liver disease/acute alcoholic hepatitis and nonalcoholic fatty liver disease/nonalcoholic steatohepatitis. Hepatology 64:955-965.

Vassallo, G., A. Mirijello, A. Ferruli, M. Antonelli, R. Landolfi, A. Gasbarrini, and G. Addolorato. 2015. Review article: Alcohol and gut microbiota-The possible role of gut microbiota modulation in the treatment of alcoholic liver disease. Aliment. Pharmacol. Ther. 41:917-927.

Wang, Y., Y. Liu, A. Sidhu, Z. Ma, C. McClain, and W. Feng. 2012. Lactobacillus rhamnosus GG culture supernatant ameliorates acute alcohol-induced intestinal permeability and liver injury. Am. J. Physiol. Gastrointest. Liver Physiol. 303:G32-G41.

Whang, T. E., H. O. Lim, and J. W. Lee. 1999. Effect of fermented (Oenanthe stolonifera DC) extract on the activity of enzymes related to liver function of alcohol-administered rats and mice. Hanguk Yakyong Changmul Hakhoe Chi 7:107-114.

Zhao, L., Y. Jiang, Y. Ni, T. Zhang, C. Duan, C. Huang, Y. Zhao, L. Gao, and S. Li. 2017. Protective effects of Lactobacillus plantarum $\mathrm{C} 88$ on chronic ethanol-induced liver injury in mice. J. Funct. Foods 35:9.

\section{ORCIDS}

Wonyong Kim @ https://orcid.org/0000-0001-9649-3919 